\title{
Bayesian Analysis Supports Atezolizumab plus Bevacizumab in Unresectable Hepatocellular Carcinoma
}

\author{
Alessandro Rizzo Angela Dalia Ricci Giovanni Brandi \\ Medical Oncology, IRCCS Azienda Ospedaliero-Universitaria di Bologna, Bologna, Italy
}

Dear Editor,

Of all the recent advances being made in unresectable hepatocellular carcinoma (HCC), the survival improvement from the use of atezolizumab-bevacizumab is probably the most striking [1]. In fact, the results of the IMbrave150 trial have being a historical step forward in this setting, with atezolizumab-bevacizumab representing the first treatment to improve overall survival and progression-free survival beyond the standard of care sorafenib in treatment-naïve patients [2]. However, only a proportion of HCC patients benefit from atezolizumab-bevacizumab, highlighting the need for a better understanding of how immune-based combinations and HCC could interact [3]. At the same time, although several potential predictive biomarkers have been explored, none have been prospectively validated.

In the current systemic review and network metaanalysis (NMA) conducted by Vogel and colleagues, the authors pulled together 9 clinical trials for a total of 3,897 patients with locally advanced or metastatic unresectable HCC [4]. According to the results of the NMA, Vogel et al. [4] suggested that atezolizumab-bevacizumab reported the most consistent overall survival benefit compared to other treatment options. Bayesian NMA was used to optimize data extrapolation and to allow a comparison between different treatments when no direct comparative study was available and to obtain more precise effect es-

karger@karger.com www.karger.com/lic

Karger!

bOPEN ACCESS
(C) 2021 The Author(s).

Published by S. Karger AG, Basel

This is an Open Access article licensed under the Creative Commons Attribution-NonCommercial-4.0 International License (CC BY-NC) (http://www.karger.com/Services/OpenAccessLicense), applicable to the online version of the article only. Usage and distribution for commercial purposes requires written permission. timated by jointly considering indirect and direct comparisons [5]. Vogel and colleagues used well-accepted and rigorous methods to compare evidence across clinical trials, also reporting and acknowledging some limitations, such as different median follow-up time among studies and limited or inconsistent reporting of some outcomes. Nonetheless, we think some methodological issues would deserve discussion.

First, the included trials presented heterogeneous study design, and the statistics quantifying the NMA heterogeneity were not reported. Second, although the authors reported that risk of bias was generally low across trials, lack of study blinding in 7 of 9 trials raises concerns, and in addition, risk of bias could not be assessed for CheckMate 459 [4]. Third, if certainly Bayesian NMA represents a powerful method that allows the sharing of information across different clinical trials, cross-trial comparisons may only partially compare adverse events and safety profiles [5]. Last, it is also important to consider that Bayesian NMA, similarly to pairwise meta-analysis, could be associated with the inflation of specific errors, such as type 1 (false positive) and type 2 (false negative) errors. Since type 1 and type 2 errors have been suggested to play a key role in the validation of true-negative and true-positive findings in meta-analysis, this point should be carefully considered. 
In our opinion, the authors are to be commended for this NMA aimed at investigating a fundamental topic in unresectable HCC, and this study provides further evidence supporting the combination of atezolizumab plus bevacizumab in this setting. At the same time, Bayesian NMA presents some limitations that should be kept in mind and does not present the statistical power to replace head-to-head clinical trials comparison. Based on these premises, we think that the current NMA further emphasizes the need for large-scale randomized trials aimed at comparing active treatments with each other in unresectable HCC.

Atezolizumab-bevacizumab has become the new benchmark for front-line treatment in unresectable HCC [1-4]; on the horizon, there is a host of phase III randomized clinical trials of novel combinations including immune checkpoint inhibitors combined with other antiangiogenic agents as well as PD-1 or PD-L1 inhibitors plus CTLA-4 inhibitors. The results of these studies are highly awaited and have the potential to further modify the first-line treatment scenario of this challenging malignancy.

\section{Conflict of Interest Statement}

The authors have no conflicts of interest to declare.

\section{Funding Sources}

No funding was received.

\section{Author Contributions}

All authors contributed equally to this work.

\section{References}

Atezolizumab-Bevacizumab in HCC
1 Kudo M. A new era in systemic therapy for hepatocellular carcinoma: atezolizumab plus bevacizumab combination therapy. Liver Cancer. 2020 Apr;9(2):119-37.

2 Finn RS, Qin S, Ikeda M, Galle PR, Ducreux M, Kim TY, et al. IMbrave150 investigators. Atezolizumab plus bevacizumab in unresectable hepatocellular carcinoma. N Engl J Med. 2020 May 14;382(20):1894-905.

3 Kudo M. Sequential therapy for hepatocellular carcinoma after failure of atezolizumab plus bevacizumab combination therapy. Liver Cancer. 2021 Apr;10(2):85-93.
4 Vogel A, Rimassa L, Sun HC, Abou-Alfa GK, El-Khoueiry A, Pinato DJ, et al. Comparative efficacy of atezolizumab plus bevacizumab and other treatment options for patients with unresectable hepatocellular carcinoma: a network meta-analysis. Liver Cancer. 2021 Jun; 10(3):240-8.

5 Higgins JP, Jackson D, Barrett JK, Lu G, Ades AE, White IR. Consistency and inconsistency in network meta-analysis: concepts and models for multi-arm studies. Res Synth Methods. 2012 Jun;3(2):98-110. 